\title{
Genotipificación del melanoma en Colombia
}

\author{
Genotyping melanoma in Colombia
} \author{
Andrés Felipe Cardona $a^{1,2, \S}$ \\ 'Grupo Oncología Clínica y Traslacional, Instituto de Oncología, Fundación Santa Fe de Bogotá (Bogotá, Colombia). \\ 2Fundación para la Investigación Clínica y Molecular Aplicada del Cáncer (FICMAC); Investigador asociado ONCOLGroup. \\ ${ }^{3}$ Departamento de Patología, Fundación Universitaria Ciencias de la Salud (FUCS), Hospital de San José (Bogotá, Colombia). \\ ${ }^{4}$ Instituto de Genómica Humana, Pontificia Universidad Javeriana (Bogotá, Colombia). \\ Departamento de Oncologia, Hospital Universitario de Santander (Bucaramanga, Colombia). \\ Departamento de Patologia, Universidad Nacional de Colombia (Bogotá, Colombia). \\ Departamento de Hematología y Oncologia, Oncologos de Occidente (Armenia, Colombia). \\ (BOSCAL) (Bucaramanga, Colombia). \\ ${ }^{10}$ Departamento de Hematología y Oncología, Clínica Astorga/SOMA (Medellín, Colombia). \\ "Departamento de Oncología, Unidad de Cancerologia, Hospital Pablo Tobón Uribe (Medellín, Colombia). \\ ${ }_{12}^{2}$ Departamento de Oncología, Instituto Nacional de Cancerología (INCAN) (México, México D.F.). \\ ${ }^{13}$ Departamento de Oncología, Clínica Colsanitas (Bogotá, Colombia). \\ ${ }^{14}$ Sección Cirugía de Seno y Tejidos Blandos, Clínica Colsanitas (Bogotá, Colombia). \\ sEstos autores contribuyeron de forma equitativa en el estudio.
}

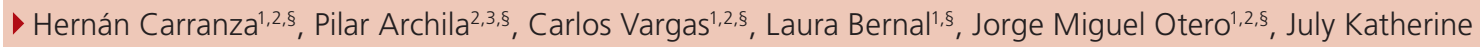

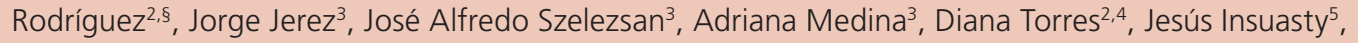
Orlando Ricaurte ${ }^{6}$, Diego Lopera ${ }^{7}$, Alejo Jiménez ${ }^{8}$, Carlos Rojas ${ }^{9}$, Mauricio Lema10, Isabel Durango ${ }^{11}$, Leonardo Rojas², Andrés Yepes ${ }^{11}$, Martha Lucía Celiz², Gustavo Rojas ${ }^{7}$, Ricardo Duarte ${ }^{13}$, Rodolfo Gómez ${ }^{8}$, Elías Quintero ${ }^{14}$,

\section{Resumen}

Introducción: El melanoma presenta una elevada heterogeneidad clonal que sigue un patrón geográfico. Con el advenimiento de los inhibidores específicos del BRAF, se han realizado múltiples esfuerzos para explorar el genotipo de estas neoplasias a nivel mundial, documentando las alteraciones puntuales V600E y V600K en cerca del $50 \%$ de los afectos nacidos en los Estados Unidos, Europa occidental y Australia. Pocos estudios han evaluado la presencia de alteraciones en el BRAF y en el KIT en población hispana, encontrando una frecuencia entre el 39 y $77 \%$, y 0\%, respectivamente.

Materiales y métodos: Se exploró en 81 pacientes la presencia de mutaciones en el BRAF (V600E/V600K), NRAS (exones 1 y 2) y CKIT (exones 9 y 11) usando técnicas de secuenciación y RT-PCR (COBAS), previa confirmación de la histología y microdisección.

Resultados: El promedio de edad fue de 53 años $(D E \pm 14,5)$ y el $59 \%$ tenía más de 50 años al momento del diagnóstico; 47 casos (58\%) eran mujeres y el resto hombres. Según el origen del melanoma, el 39,5\% presentaba tumores originados en piel crónicamente expuesta a la luz solar, el 19,8\% no fueron tipificables, el 19,8\% tenía neoplasias lentiginosas acrales, el 7,4\% tenía primarios de mucosas y el 1,2\% tumores uveales. En general, la representación tumoral en el tejido embebido en parafina fue buena ( $80 \%)$, el sitio para la toma de la muestra fue en la gran mayoría la piel (42\%), los ganglios (26\%) y el pulmón (9,8\%). En el $70 \%$ de los casos, el estado tumoral fue mayor que 3 y en el $30 \%$ fue imposible obtener la información concerniente a este ítem. La frecuencia de mutaciones en BRAF fue $24,7 \%(n=20)$, para cKIT fue del $4,9 \%(n=4)$ y para NRAS fue del 6,1\% $(n=5)$. El 69,4\% (36 casos evaluables) tuvo un KI67 mayor al 20\%, hallazgo que fue mayor en los pacientes con lesiones originadas en piel crónicamente expuesta $(p=0,052)$ y entre los mutados del BRAF $(p=0,048)$.

Conclusiones: El perfil mutacional de los pacientes colombianos con melanoma es diferente al descrito previamente en la literatura, en especial para BRAF. Estos hallazgos son concordantes con una mayor prevalencia de lesiones originadas en mucosas y del tipo lentiginoso acral.

Palabras clave: melanoma, genotipificación, mutación, oncogén, BRAF.

\begin{abstract}
Introduction: Melanoma has high clonal heterogeneity which follows a geographical pattern. The advent of therapy involving BRAF-specific inhibitors has enabled many efforts at exploring these neoplasias' genotype around the world, documenting V600E and V600K alterations in about $50 \%$ of affected patients born in the USA, Western Europe and Australia. Few studies have evaluated the presence of alterations in the BRAF and KIT in the Hispanic population, finding a frequency between 39 and $77 \%$, and $0 \%$, respectively.
\end{abstract}


Materials and methods: 81 patients were explored for BRAF (V600E/V600K), NRAS (exons 1 and 2) and cKIT (exons $9,11,13$ and 17) mutations using sequencing and RT-PCR (COBAS) techniques, following confirmation of histology and micro-dissection.

Results: The patients were aged 53 years old on average $(S D \pm 14,5)$ and $59 \%$ were older than 50 when diagnosed; 47 cases $(58 \%)$ were female. When ascertaining the origin of the patients' melanomas $39,5 \%$ of the tumours were found in skin which had been chronically exposed to sunlight, 19,8\% could not be typed, 19,8\% were acral-lentiginous melanomas, 7,4\% were primary mucosal melanomas and 1,2\% were uveal tumours. Tumour representation in paraffin-embedded tissue was good $(80 \%)$, the site from which the sample was taken was usually the skin $(42 \%)$, lymph nodes (26\%) and the lungs $(9.8 \%)$. Tumour stage was greater than 3 in $70 \%$ of the cases; however, information concerning this item could not be obtained for $30 \%$ of the patients. BRAF mutation frequency was $24,7 \%(n=20), 4,9 \%(n=4)$ for CKIT and $6,1 \%(n=5)$ for NRAS. 69,4\% (36 evaluable cases) had greater than $20 \% \mathrm{KI} 67$; this finding was greater in patients suffering lesions in chronically-exposed skin $(p=0,052)$ and in those carrying a BRAF mutation $(p=0,048)$.

Conclusions: The mutational profile of Colombian melanoma patients reported in this study differ with that described previously, especially for BRAF; however, such findings did agree with greater prevalence of mucosal and acral-lentiginous lesions.

Key words: melanoma, genotipying, mutation, oncogen, BRAF.

\section{Introducción}

El melanoma es la forma más agresiva del cáncer de piel y una de las enfermedades oncológicas con mayor letalidad; aunque representa solo el $4 \%$ de los tumores malignos de este origen, es responsable del 75 al 90\% de las muertes por esta causa'. Su incidencia y mortalidad a nivel mundial está aumentando a una tasa superior a la de cualquier otro tumor; entre los años 1985 y 2005, el riesgo de desarrollar un melanoma a lo largo de la vida pasó de ser 1/150 a 1/37 en los hombres y de 1/600 a 1/56 en las mujeres. Por esta proyección, se estima una duplicación de los casos incidentes cada 10 a 20 años $^{1}$. Actualmente, se diagnostican 75.000 casos nuevos y 9.000 muertes por melanoma a nivel mundial cada año ${ }^{2,3}$. La mayoría de los pacientes con enfermedad temprana presentan recurrencia local o a distancia a pesar de la cirugía, radiación y/o inmunoterapia, con una supervivencia global a cinco años cercana al $40 \%{ }^{4}$. Al desarrollarse las metástasis, el melanoma se torna refractario a la mayoría de las estrategias terapéuticas convencionales, manifestando respuestas inferiores al 10 al 15\%, de forma que la supervivencia global a cinco años en este escenario disminuye al $15 \% \%^{5-7}$. Según datos de Globocan para el 2008 en Colombia, la incidencia y mortalidad por melanoma fue de 1,9 y 0,5 casos por cada 100.000 habitantes, respectivamente; esto significa que la enfermedad afecta a 855 personas cada año y es la causa de muerte en 215 de ellas en el mismo período.

Durante la última década, la investigación se ha concentrado en determinar el perfil genómico que favorece el desarrollo del melanoma, al igual que entender su compleja heterogeneidad clonal para encontrar nuevas estrategias de manejo ${ }^{8}$. Se han identificado diversos oncogenes conductores que siguen una presentación variable, dependiente de la localización y de la epidemiología molecular. La alteración genética más frecuente ocurre en BRAF, un protooncogén localizado en el cromosoma 7 que codifica una serina/treonina proteinquinasa perteneciente a la superfamilia RAF, componente de la vía de señalización de las MAP quinasas $^{9}$. Se han descrito distintas mutaciones puntuales, pero la más frecuente (85-92\%) ocurre en el exón 15 y conduce a la sustitución de un ácido glutámico por valina en el codón $600(\mathrm{~V} 600 \mathrm{E})^{10}$. La segunda mutación más común (V600K - lisina por valina) representa un 10 al 15\% de las modificaciones del gen. Estas mutaciones conducen a una activación anormal de BRAF (130 a 700 veces mayor que la actividad normal) que a su vez incrementa el potencial de transformación de los melanocitos (estimulando la proliferación celular, la supervivencia, la motilidad y la angiogénesis) ${ }^{11}$. Múltiples estudios epidemiológicos han determinado que las mutaciones del BRAF están presentes hasta en el 66\% de los melanomas, siendo más frecuentes en pacientes menores de 55 años, en las lesiones con extensión superficial y en los tumores originados en sitios de la piel expuestos de forma intermitente al sol' ${ }^{12}$.

Por otra parte, también se han documentado mutaciones en el NRAS (10 al 30\% $)^{13}$, el cual codifica para una proteína con actividad GTPasa que interviene tempranamente en la vía MAP quinasa y en PI3K/AKT. Estas variaciones involucran principalmente los codones 12,13 y 61, y se encuentran con mayor frecuencia en los melanomas de los sitios con exposición importante a la radiación ultravioleta ${ }^{14}$. En otra fracción (17-23\%), se han evidenciado alteraciones en KIT, gen que codifica para un receptor transmembrana del tipo $\mid I^{16,17}$ que, al unirse a su ligando, activa diferentes vías de señalización 
en los melanomas de tipo lentiginoso acral y en los primarios de mucosas. La mutación dominante se presenta en el exón 11, donde se sustituye una leucina por una prolina en el codón 576, y con menor frecuencia en los exones 13,17 y $18^{18-22}$. Por último, la evidencia muestra que estas tres mutaciones (BRAF, NRAS y c-KIT) son mutuamente excluyentes ${ }^{15}$.

Conocer el genotipo del melanoma ha permitido la implementación de nuevas terapias dirigidas. El vemurafenib, un inhibidor selectivo del BRAF, mostró una tasa de respuesta del $48 \%$ en el estudio BRIM3, así como una mejoría significativa en la supervivencia libre de progresión (SLP) (HR 0,26, IC95\% 0,20-0,33; $\mathrm{p}<0,0001)$ y global (SG) (HR 0,37, IC95\% 0,26-0,55; $p<0,0001)$ versus la dacarbacina ${ }^{23,24}$. Paralelamente, algunos inhibidores de tirosinquinasa, particularmente el imatinib, han mostrado ser efectivos en melanomas con mutaciones de KIT ${ }^{20-22}$. Estos y otros avances, entre los que cabe destacar a los inhibidores MEK, antiCTLA-4 y anti-PD1/PDL1, han revolucionado el manejo de pacientes con melanoma metastásico ${ }^{25,26}$.

El éxito de la terapia dirigida ha impulsado la realización de un sinnúmero de estudios de genotipificación del melanoma a nivel mundial; la literatura existente sugiere una frecuencia altamente variable de las mutaciones descritas según la localización geográfica, las características étnicas, la exposición a rayos ultravioleta y los tipos histológicos ${ }^{27-30}$. La mayoría de los trabajos se han llevado a cabo en población caucásica de los Estados Unidos, Europa y Australia ${ }^{26,27}$, y unos pocos se han publicado en Asia28-30 y América Latina ${ }^{31-33}$.

El objetivo de este estudio fue evaluar la presencia de mutaciones en el BRAF, NRAS y c-KIT, en una serie de pacientes colombianos con melanoma, al igual que valorar su relación con las distintas características clínicas y su impacto sobre múltiples desenlaces.

\section{Materiales y métodos}

\section{Población}

El estudio incluyó 81 adultos con diagnóstico histológico confirmado de melanoma ingresados en la Plataforma de Investigación Meta-Melanoma de la Fundación para la Investigación Clínica y Molecular Aplicada del Cáncer (MM-FICMAC). Todos los especímenes fueron evaluados por dos pares de patólogos entrenados (AM y OR y JJ y JAS), quienes seleccionaron tres cortes de $5 \mathrm{~mm}$ de espesor fijados en formalina del tejido embebido en parafina. Estas secciones fueron llevadas a microdisección (PA) previa identificación de las áreas enriquecidas por tumor con mínima necrosis. Para la selección, el registro de los pacientes debía incluir al menos la siguiente información: diversas características clínicas (identificación, edad, sexo, tipo de melanoma, estado tumoral estratificado por el American Joint Committee on Cancer Staging $-7^{\text {th }}$ edition ${ }^{34}$, presencia de ulceración, fecha de diagnóstico, último control y muerte), datos sobre la patología (representatividad tumoral, origen del tejido, Ki67, Breslow, nivel de Clark), el genotipo (evaluación secuencial para BRAF, NRAS y CKIT) y un desenlace (supervivencia global). Todos los pacientes consintieron la evaluación genómica al igual que la captura de la información clínica.

\section{Extracción y amplificación del ADN}

Las células microdisecadas se incubaron durante 72 horas a $37^{\circ} \mathrm{C}$ en 50 a $100 \mathrm{ml}$ de un tampón para lisis (10 mmoles/l de Tris- $\mathrm{HCl}, \mathrm{pH} 1 / 4$ 8,0; $1 \%$ de Tween-20; $1 \mathrm{mmol} / \mathrm{l}$ de EDTA; y el 0,04\% proteinasa $\mathrm{K}$ ). Después de una centrifugación a alta velocidad de cinco minutos, la muestra se calentó a $95^{\circ} \mathrm{C}$ durante 8 minutos para inactivar la proteinasa K. La PCR se realizó usando el kit de reactivos de GeneAmp Gold PCR (Applied Biosystems, Life Technologies, Carlsbad, CA, Estados Unidos). Los exones de cKIT se amplificaron en reacciones singleplex. La PCR anidada para los exones de CKIT (primera y segunda amplificaciones) se llevó a cabo en volúmenes de reacción de $20 \mu \mathrm{l}$, incluyendo $2 \mu$ de la plantilla (template) de ADN. Los primers y condiciones para el exón 2 de NRAS, el exón 15 de BRAF y los exones 9, 11, 13 y 17 del CKIT fueron proporcionados por Roche Diagnostics y se enumeran a continuación:

\begin{tabular}{|c|c|}
\hline NRAS exón 2 & $\begin{array}{l}\text { Forward: } 5^{\prime} \text {-CCCCTTACCCTCCACAC-3' } \\
\text { Reverse: } 50 \text {-AGGTTAATATCCGCAAATGAC- } 3^{\prime} \\
95^{\circ} \mathrm{C} 45 \text { segundos, } 55^{\circ} \mathrm{C} 45 \text { segundos, } 72^{\circ} \mathrm{C} 60 \text { segundos; } \\
40 \text { ciclos de cloruro de magnesio; } 1,5 \mathrm{mmol} / \mathrm{l}\end{array}$ \\
\hline BRAF exón 15 & $\begin{array}{l}\text { Forward: } 5^{\prime} \text {-TCATAATGCTTGCTCTGATAGGA-3' } \\
\text { Reverse: } 5^{\prime} \text {-GGCCAAAAATTAATCAGTGGA-3' } \\
94^{\circ} \mathrm{C} 30 \text { segundos, } 57^{\circ} \mathrm{C} 60 \text { segundos, } 72^{\circ} \mathrm{C} 60 \text { segundos, } \\
40 \text { ciclos; cloruro de magnesio } 1,5 \mathrm{mmol} / /\end{array}$ \\
\hline $\begin{array}{l}\text { KIT exones } 9,11 \text {, } \\
13 \text { y } 17\end{array}$ & $\begin{array}{l}\text { Exón } 11 \\
\text { Forward: 5'-TGTTCTCTCTCCAGAGTGCTCTAA-3' } \\
\text { Reverse: 5'-AAACAAAGGAAGCCACTGGA-3' } \\
\text { Exón } \mathbf{1 3} \\
\text { Forward: 5'-CATCAGTTTGCCAGTTGTGC-3' } \\
\text { Reverse: 5'-AGCAAGAGAGAACAACAGTCTGG-3' } \\
\text { Exón 17 } \\
\text { Forward: 5'-TCATTCAAGGCGTACTTTTG-3' } \\
\text { Reverse: 5'-TCGAAAGTGGAACTAAAAATCC-3' } \\
\text { Exón } \mathbf{9} \\
\text { Forward: 5'-CATTTCAGCAACAGCAGCAT-3' } \\
\text { Reverse: 5'-CAAGGAAGCAGGACACCAAT-3' }\end{array}$ \\
\hline
\end{tabular}


Los productos de PCR fueron purificados usando el kit QIAquick PCR Purification (Qiagen Inc., Valencia, CA, EE. UU.) de acuerdo con las instrucciones del fabricante. La secuenciación de los productos de la PCR en ambas direcciones se realizó utilizando un Prism 3100 Genetic Analyzer ADN ABI (Applied Biosystems, Life Technologies, Carlsbad, CA, EE. UU.), empleando el Big Dye v.3.1 dye terminator chemistry. Los resultados del cromatograma se interpretaron de forma independiente por dos investigadores (JKR y AFC) y todas las mutaciones se identificaron en ambas cadenas. Los resultados para el BRAF fueron confirmados usando el Cobas 4800 BRAF V600 Mutation Test (Roche Molecular Systems Inc., Branchburg, New Jersey, EE. UU.).

\section{Estadística}

Los resultados se obtuvieron y describieron por medio de la determinación de frecuencias absolutas, relativas, medidas de tendencia central y de dispersión. El cruce de variables se efectuó con tablas de contingencia sometidas a pruebas de dependencia y asociación usando el estadístico Chi cuadrado $\left(X^{2}\right)$ o el test exacto de Fisher cuando fue necesario. Para todos los casos, el nivel de significancia fue $p<0,05$. Las estimaciones de supervivencia se hicieron utilizando el modelo no paramétrico del límite del producto (método de Kaplan-Meier), y sus funciones se compararon mediante la prueba Log-rank. Con el fin de valorar los factores que influyeron sobre la mortalidad, se llevó a cabo un análisis multivariado empleando el modelo de riesgo proporcional (Cox).

\section{Resultados}

La edad de los pacientes estuvo entre 22 y 84 años (media 53,8, DE $\pm 14,5$ ), de los cuales, 47 (58\%) fueron mujeres y 34 (42\%) hombres. La representación tumoral dentro del tejido embebido en parafina fue buena (> 50\%) en el $80,3 \%$ de los casos, moderada (30-50\%) en el 7,4\% y mínima o escasa ( $<30 \%$ ) en el $9,8 \%$. En cuanto al origen del melanoma, el 39,5\% presentó tumores originados en piel crónicamente expuesta a la luz solar, el 19,8\% no fueron tipificables, el 19,8\% fueron neoplasias lentiginosas acrales, el 7,4\% primarios de mucosas y el 1,2\% melanomas uveales. Referente al estado tumoral, el 2,5\% tuvo un estado II; el 23,4\%, estado III; y el 46,9\%, estado IV (en el 27,2 no fue posible obtener esta información). En 59 casos, se evaluó la presencia de ulceración, que se documentó en el $22 \%(n=13)$. En 47 y 41 pacientes, se recolectaron los datos sobre el Breslow y el Clark, respectivamente; el $98 \%(n=46)$ tuvo un Breslow mayor que $2,1 \mathrm{~mm}$ y el $58 \%(n=24)$ un nivel de Clark superior a 2 (tabla 1$)$. La figura 1 incluye las variantes histológicas más representativas del estudio.

Tabla 1. Clasificación de los melanomas según el Breslow y Clark

\begin{tabular}{|l|c|c|}
\hline \multicolumn{2}{|c|}{ Variable } & $\begin{array}{c}\text { Frecuencia } \\
(\mathbf{n})\end{array}$ \\
\hline Breslow & 1 & 2,1 \\
\hline $1-2 \mathrm{~mm}$ & 32 & 68,1 \\
\hline $2,1-4 \mathrm{~mm}$ & 14 & 29,8 \\
\hline$>4 \mathrm{~mm}$ & 34 & 100,0 \\
\hline No hay dato & & \\
\hline Clark* & 4 & 9,8 \\
\hline Nivel II & 13 & 31,7 \\
\hline Nivel III & 18 & 43,9 \\
\hline Nivel IV & 6 & 14,6 \\
\hline NivelV & 40 & 100,0 \\
\hline No hay dato & & \\
\hline
\end{tabular}

* Nivel II: Invasión de la dermis papilar que no alcanza la interfase papilorreticular de la dermis. Nivel III: Invasión que ocupa la dermis papilar y limita con la dermis reticular pero no la penetra. Nivel IV: Invasión de la dermis reticular pero no del tejido celular subcutáneo. Nivel V: Invasión a través de la dermis reticular al tejido celular subcutáneo.

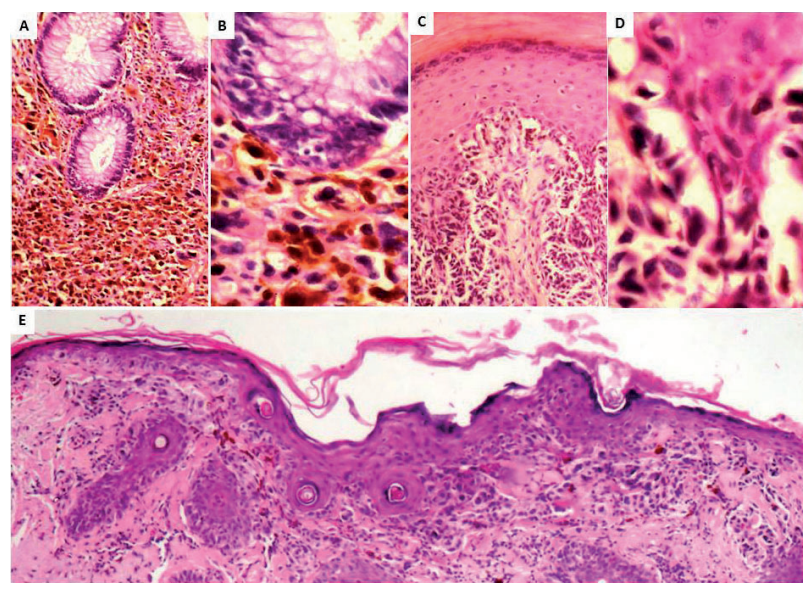

Figura 1. A y B. Melanoma primario de mucosas (colon) hematoxilina y eosina 100x y 400x; C y D. Melanoma lentiginoso acral (miembro inferior) hematoxilina y eosina $100 x$ y $400 x$. E. Lentigo maligno hematoxilina y eosina $40 x$.

Se encontraron mutaciones en el oncogén BRAF en el $24,7 \%(n=20)$, en todos los casos, correspondiendo con la alteración $\mathrm{V} 600 \mathrm{E}$. El índice de mutaciones en el NRAS fue del 6,1\% (4 en el exón 1 y 1 en el exón 2) y el porcentaje de alteraciones en el KIT fue del 4,9\% (una en el 9 -N463S-y 3 en el exón 11 -L576P-) (figura 2). Al seleccionar únicamente los melanomas acrales, la proporción de mutaciones en el KIT fue del 33\%. 


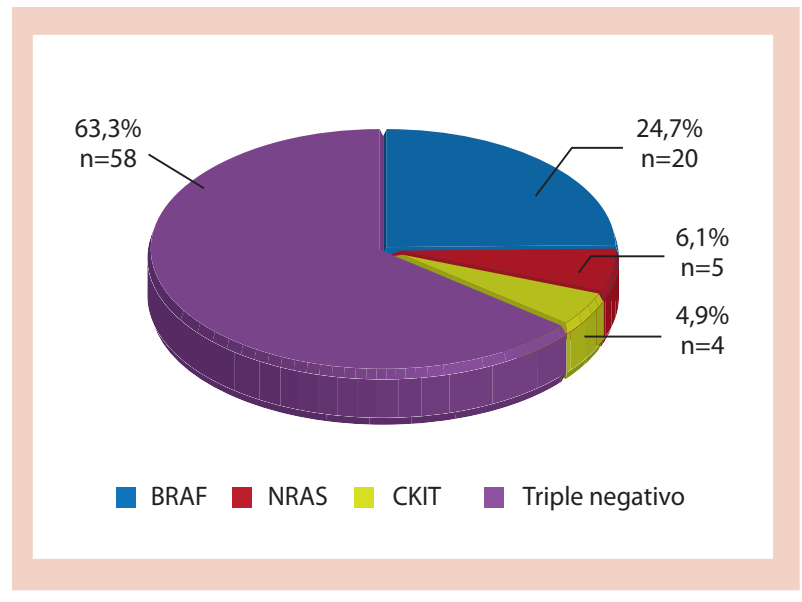

Figura 2. Distribución de las mutaciones en pacientes con melanoma tipificados en Colombia.

La asociación entre las diferentes mutaciones y cada una de las características clínicas dominantes está resumida en la tabla 2. La posibilidad de documentar mutaciones en el BRAF fue mayor en los tumores primarios en comparación con el tejido obtenido de metástasis $(p=0,034)$. También, se encontró asociación entre la localización del tumor y las mutaciones en el KIT, siendo estas más frecuentes en los melanomas de tipo lentiginoso acral $(p=0,044)$. No se halló relación entre las mutaciones del NRAS y otras variables. El 69,4\% tuvo un Ki67 mayor al 20\%, hallazgo predominante entre los pacientes con lesiones originadas en la piel crónicamente expuesta $(p=0,052)$ y entre los mutados del BRAF $(p=0,048)$.

Se obtuvieron datos de la supervivencia global (SG) en 33 pacientes, para los que la mediana fue 18 meses (IC 95\% 14,4-21,5) (figura 3). Al valorar la mediana para la SG dependiente del sexo, se encontró una tendencia positiva a favor de la población masculina (29 meses [IC 95\% 19,8-38,1] vs. 16 meses [IC 95\% 11,5-20,4];

Tabla 2. Asociación del genotipo con algunas características demográficas e histopatológicas

\begin{tabular}{|l|c|c|c|}
\hline \multicolumn{1}{|c|}{ Variable } & BRAF & c-KIT & NRAS \\
\hline Edad & $\begin{array}{c}\text { No } \\
p=0,19\end{array}$ & $\begin{array}{c}\text { No } \\
p=0,52\end{array}$ & $\begin{array}{c}\text { No } \\
p=0,38\end{array}$ \\
\hline Sexo & $\begin{array}{c}\text { No } \\
p=0,16\end{array}$ & $\begin{array}{c}\text { No } \\
p=0,60\end{array}$ & $\begin{array}{c}\text { No } \\
p=0,60\end{array}$ \\
\hline Tipo de melanoma & $\begin{array}{c}\text { No } \\
p=0,10\end{array}$ & $\begin{array}{c}\text { Sí } \\
p=0,04\end{array}$ & $\begin{array}{c}\text { Sí } \\
p=0,65\end{array}$ \\
\hline Origen del tejido & $\begin{array}{c}\text { Sí } \\
p=0,034\end{array}$ & $\begin{array}{c}\text { No } \\
p=0,27\end{array}$ & $\begin{array}{c}\text { No } \\
p=0,46\end{array}$ \\
\hline Estado tumoral & $\begin{array}{c}\text { No } \\
p=0,27\end{array}$ & $\begin{array}{c}\text { No } \\
p=0,27\end{array}$ & $\begin{array}{c}\text { No } \\
\text { Sín }\end{array}$ \\
\hline Ki67 & $\begin{array}{c}\text { Nín } \\
p=0,04\end{array}$ & $\begin{array}{c}\text { No } \\
p=0,58\end{array}$ \\
\hline
\end{tabular}

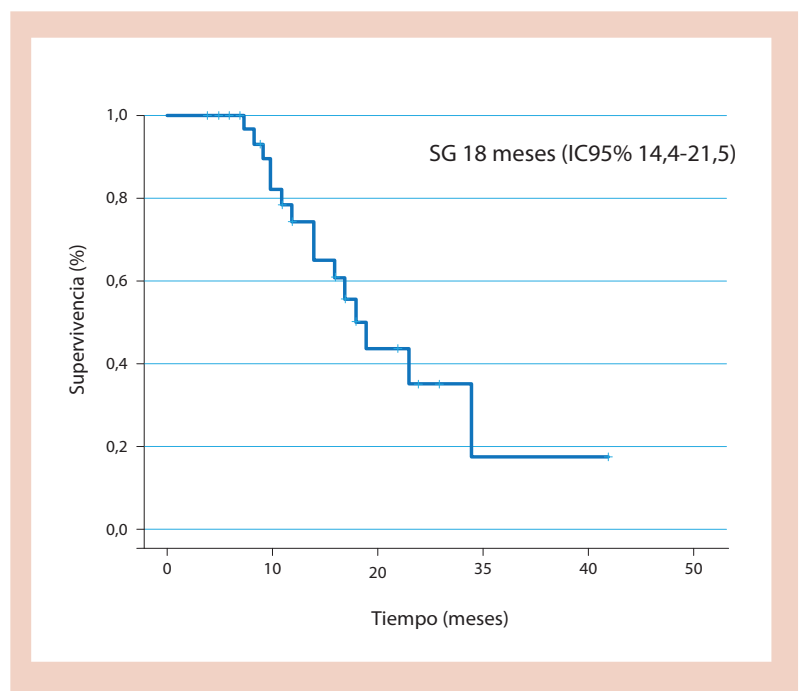

Figura 3. Supervivencia global.

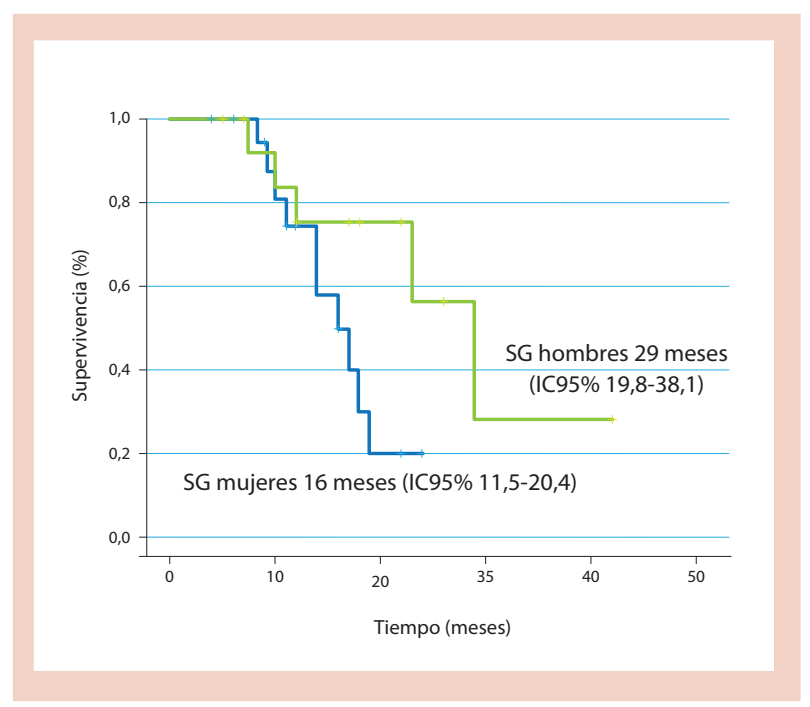

Figura 4. Supervivencia global según el sexo.

$p=0,087$ ) (figura 4). La SG no se correlacionó con la edad $(p=0,89)$ ni con el tipo de melanoma $(p=0,56)$ (figura 5). La mediana para la SG fue de 17 meses (IC 95\% 7,3-26,6) en el grupo de sujetos con lesiones originadas en la piel crónicamente expuesta, 23 meses (IC 95\% 9,6-36,3) para los que tuvieron lesiones de piel sin especificar, 15,8 meses para los lentigoas acrales (IC 95\% $12,1-19,5)$ y 28 meses para los melanomas originados en las mucosas (IC 95\% 8,5-47,4). Se demostró una asociación positiva entre el estado de la enfermedad y la SG ( $p$ $=0,03$ ), siendo inferior para los pacientes con metástasis (figura 6). No se halló relación alguna entre la SG y el genotipo basal (BRAF $p=0,79$, c-KIT $p=0,23$, NRAS $p$ $=0,49)$, el Ki67 $(p=0,42)$, la presencia de ulceración ( $p$ $=0,37)$, el Breslow $(p=0,35)$ y el Clark $(p=0,76)$. 


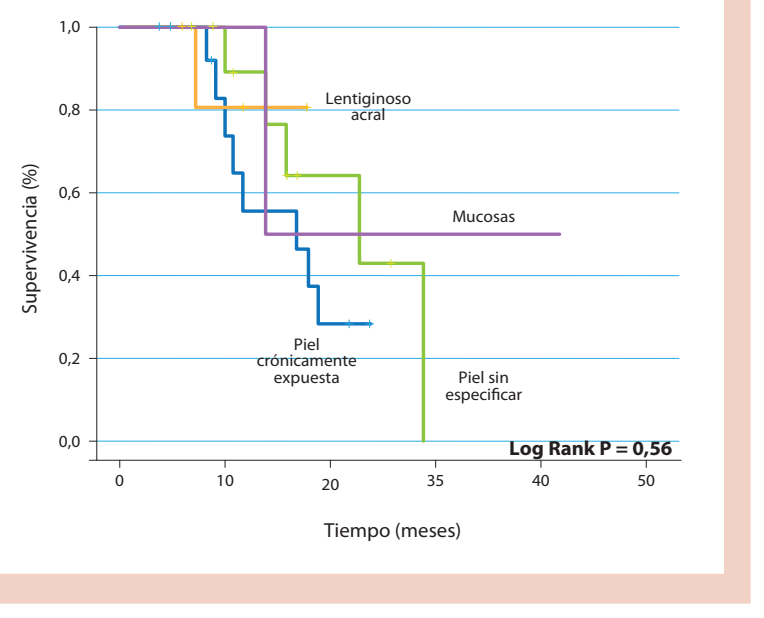

Figura 5. Supervivencia global según el tipo de melanoma.

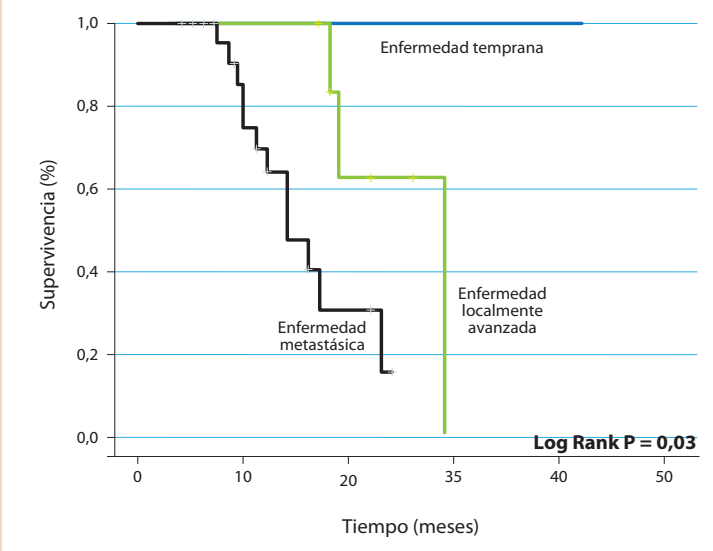

Figura 6. Supervivencia global según estado de la enfermedad.

Al realizar el análisis multivariado, utilizando el modelo de Cox, se encontró que el estado tumoral fue la única variable que modificó el curso de la supervivencia ( $p=0,03$; IC 95\% 1,17-23). Al cruzar el Ki67 (variable recodificada en $<20 \%$ y $>20 \%$ ) versus el estado tumoral agrupado, se documentó una tendencia hacia la mayor proliferación celular entre los tumores metastásicos $(p=0,068)$.

\section{Discusión}

El presente estudio es el primer esfuerzo para genotipificar el melanoma en Colombia; también constituye una evaluación ampliada del genoma de esta enfermedad en América Latina, incluyendo la evaluación de alteraciones en BRAF, KIT y NRAS.
La tasa de mutaciones en el BRAF encontrada en la serie de pacientes de Colombia $(24,7 \%)$ fue notablemente inferior a la reportada en la población caucásica (alrededor del 66\%) y en los pocos estudios realizados en otras regiones de América Latina; un estudio efectuado en Brasil que incluyó 96 pacientes reportó un $39 \%$ y otro ejecutado en Uruguay (27 pacientes) informó un 78\% ${ }^{31-33}$; por otra parte, nuestros datos fueron similares a los hallados en diversas poblaciones asiáticas (Japón, 101 pacientes, 26,7\%; China, 432 pacientes, 25,5\%)28-30. Dicha frecuencia fue mayor al seleccionar únicamente las lesiones originadas en la piel $(32,7 \%)$. Aunque en múltiples ocasiones se ha descrito una fuerte asociación entre la presencia de la mutación en BRAF y el origen del tumor en sitios de la piel expuestos de forma intermitente al sol ${ }^{14}$, no hemos observado asociación entre el estado del oncogén y la localización del tumor primario en esta serie. A pesar de que varios estudios soportan esta hipótesis, otros han descrito que las mutaciones de BRAF están distribuidas uniformemente entre los diferentes histotipos ${ }^{35}$.

Una investigación llevada a cabo por Mar y colaboradores reportó una tasa media de mutaciones en el melanoma de 21 por $\mathrm{Mb}$ en las lesiones generadas en la piel con daño solar severo, versus 3,8 por Mb en piel no expuesta $(p=0,001)^{52}$. Sin embargo, la presencia de mutaciones en el BRAF en otras neoplasias no explicadas por la radiación ultravioleta (cáncer colorrectal y tiroideo) descartan las conjeturas de una asociación germinal entre la radiación y el desarrollo de la mutación.

Recientemente, Karram y colaboradores analizaron 225 especímenes, encontrando un aumento en la incidencia de mutaciones en BRAF en el grupo de menor edad, a la vez que descartaron la dependencia entre la magnitud de la radiación UV recibida y la tasa de alteraciones genómicas en diferentes puntos de las vías de señalización dependientes de MAPK. Estos hallazgos permitieron concluir que la adquisición de la mutación de BRAF es dependiente de la duración de la exposición más que de la magnitud de la irradiación recibida9. Nuestro estudio no evidenció una asociación positiva entre la mutación del BRAF y la edad, aunque la literatura parece consistente en reportar una mayor incidencia en los menores de 50 años $^{36}$.

La totalidad de las alteraciones en BRAF encontradas en nuestra cohorte correspondieron con la modificación V600E, a pesar de realizar una doble evaluación usando 
secuenciación Sanger y RT-PCR (PCR en tiempo real) por Cobas. Llama la atención la ausencia de modificaciones en V600K y V600M, hallazgos que explican el 3\% de las lesiones ${ }^{9}$. La literatura reporta una mayor proporción de variantes distintas a la V600E en pacientes de mayor edad, donde la transversión V600K es la forma dominante. Esta alteración también se ha asociado con una mayor exposición a la luz solar en el sitio de origen del primario en comparación con lo observado en melanomas portadores de la mutación V600E. Así mismo, el genotipo V600K disminuye la supervivencia libre de progresión tras la exposición al vemurafenib; no obstante, este resultado podría ser atribuible a un sesgo de selección debido a que los pacientes con esta mutación suelen ser de mayor edad. Pese a estas diferencias en el comportamiento biológico de la enfermedad, los inhibidores del BRAF son efectivos en las mutaciones V600E y V600K ${ }^{39}$, de ahí la relevancia de identificar las alteraciones de menor frecuencia. La falla para detectar la mutación V600K podría ocasionar que un $20 \%$ de los pacientes con dominancia del BRAF (equivale hasta un 10 al $15 \%$ del total de pacientes con melanoma metastásico, proporción que podría ser más representativa en la población de sujetos de mayor edad) no tengan acceso a terapias específicas ${ }^{40}$.

En nuestro análisis, la presencia de mutaciones en BRAF fue mayor en las muestras derivadas del primario que en las lesiones metastásicas, hallazgo documentado previamente ${ }^{37}$; estos datos reflejan la importancia de la mutación del BRAF dentro de la tumorigénesis del melanoma y su posible participación en la expansión clonal durante la progresión ${ }^{37}$. Sin embargo, la información sobre el impacto de esta mutación en el pronóstico de la enfermedad no es conclusiva. Las mutaciones en BRAF se han asociado con un peor pronóstico, incluyendo una mayor tasa de ulceración, compromiso ganglionar, migración visceral y reducción de la SG ${ }^{38,41}$. En contraposición, Saroufim y colaboradores encontraron en 545 pacientes que el estado alterado del BRAF se asoció con una menor agresividad biológica ${ }^{10}$.

La frecuencia de mutaciones en el NRAS $(6,1 \%)$ fue menor en comparación con lo reportado previamente en la literatura (alrededor del 18\%) ${ }^{42}$, aunque la totalidad de las evaluaciones anteriores se hizo en caucásicos, sin que hubiere ningún reporte en población latinoamericana. La mayoría de las mutaciones se encontraron en el exón 1, y solo una en el 2, distribución similar a la descrita anteriormente ${ }^{41}$. No se halló ninguna relación entre las mutaciones en el NRAS y el pronóstico. Un metaanálisis que integró estudios publicados entre 1989 y 2010 concluyó que estas mutaciones son más frecuentes en lesiones de patrón nodular, derivadas de la piel con daño solar crónico y localizadas en las extremidades ${ }^{42}$. Adicionalmente, dos series que incluyeron más de 200 pacientes determinaron que las alteraciones en el NRAS se asocian con un mayor Breslow, Clark e índice mitótico en comparación con los tumores silvestres para este gen o con mutaciones en $\mathrm{BRAF}^{43}$; incluso, uno de estos estudios encontró que el NRAS se asoció con menor SLP y SG ${ }^{43,44}$. Otra evaluación retrospectiva mostró que la presencia de mutaciones en el NRAS constituye un factor pronóstico negativo, reportando igualmente una menor SG en pacientes mutados en comparación con tumores BRAF y NRAS silvestres $(p=0,05, H R=2,05)^{41}$. Este estudio también determinó que los pacientes con mutaciones en el NRAS o en BRAF presentan un mayor tropismo por el sistema nervioso central al momento del diagnóstico.

Un hallazgo interesante fue que la frecuencia de alteraciones en el KIT (el 4,6\% en la población global y el $33 \%$ de los melanomas acrales) fue superior a la reportada previamente. En este caso, se observó una diferencia estadísticamente significativa entre la presencia de alguna mutación en el KIT y la localización original del tumor $(p=0,044)$, siendo más frecuente en los tumores de tipo lentiginoso acral. Las mutaciones se encontraron ubicadas en algunos de los exones reportados previamente (9 y 11), sin observar alteraciones en los otros exones $(13,17,18)^{47}$. Los reportes acerca de la influencia de los cambios en KIT sobre la supervivencia son contradictorios. Kong y colaboradores describieron una disminución en la $\mathrm{SG}^{45}$, mientras que un estudio sueco no encontró la misma relación ${ }^{46}$.

Se ha postulado que el pronóstico del melanoma puede ser dependiente de la edad, siendo peor en los ancianos donde hay alteraciones constitutivas en los mecanismos inmunológicos de control o por una biología más agresiva. Varios estudios han encontrado que los mayores de 60 años tienen tumores de mayor grosor, que se presentan en estados más avanzados, particularmente en la cabeza y el cuello, donde siguen un fenotipo nodular ${ }^{28}$. La supervivencia suele ser mejor en las mujeres debido a un diagnóstico más temprano, por la presencia de tumores más delgados que se 
manifiestan generalmente en las extremidades. Un estudio de 10.538 pacientes llevado a cabo en Holanda encontró un riesgo incremental para la mortalidad entre los hombres de 2,7 veces, exceso que se mantuvo al realizar el ajuste de múltiples variables (edad, localización, Breslow, compromiso ganglionar, estado, entre otros) y que resultó comparable al de otros factores asociados al pronóstico (Breslow, ulceración, tipo histológico ${ }^{48}$. Josse y colaboradores revisaron los datos de 2.672 pacientes con melanomas tempranos encontrando una ventaja consistente e independiente de las mujeres en la SG (HR 9,7; IC95\% 0,59-0,88), incluyendo el tiempo al desarrollo de metástasis ganglionares (HR 0,70; IC95\% 9,51-0,96) y distancia (HR 0,69; IC95\% $0,59-0,81)^{54}$. Aún no se conoce la fisiopatología de este fenómeno; por ahora, se han descartado factores hormonales, puesto que no hay diferencias entre las mujeres embarazadas y no gestantes, ni entre aquellas pre o posmenopáusicas. No obstante, en nuestro estudio, encontramos una tendencia opuesta, dado el mejor pronóstico en la población masculina, con una supervivencia global que fue casi el doble que la de las mujeres (29 meses vs. 16 meses), aunque no se realizó una corrección de este resultado por otras variables que podrían crear confusión, al igual que por el limitado tamaño muestral. No se encontró asociación entre la edad y la supervivencia global.

Dada la mutua exclusividad de las tres mutaciones dominantes, la literatura soporta su evaluación secuencial. Por la mayor frecuencia de mutaciones en KIT en los melanomas acrales y originados en mucosas, este gen podría ser evaluado en paralelo con las otras dos mutaciones. Por el momento, no se recomienda evaluar mutaciones del BRAF, NRAS o del KIT en melanomas uveales, donde las alteraciones dominantes están en GNA11 (55\%) y en GNAQ (25\%) ${ }^{46}$. En el 95\% de los casos evaluados para BRAF y NRAS, el perfil mutacional se mantiene entre el primario y las metástasis ${ }^{47}$, mientras la exposición a los inhibidores selectivos genera modificaciones secundarias por selección clonal; en el caso de los pacientes mutados de BRAF, las variaciones dominantes se dan en MEK1, CRAF, y NRAS ${ }^{16}$.

Con el advenimiento de nuevas tecnologías, se han desarrollo varias plataformas de secuenciación masiva paralela - MPS (next generation sequencing o massive parallel sequencing) que permiten titular millones de fragmentos de ADN para detectar las posibles variaciones genómicas bajo una perspectiva costo-efectiva; sin embargo, la validación de sus resultados requiere un amplio conocimiento en bioinformática y largos períodos de estandarización. De igual forma, recrean errores en la evaluación masiva de muestras de melanoma que oscilan alrededor del 6 al 10\%, en especial por la limitación para la detección de variantes de un único nucleótido (SNV), eventos detectables con los métodos convencionales ${ }^{48}$.

En conclusión, el perfil mutacional de los pacientes colombianos con melanoma parece opuesto al descrito previamente en la literatura; nuestros hallazgos son concordantes con una mayor prevalencia de lesiones originadas en mucosas y del tipo lentiginoso acral. Las variaciones en la epidemiología molecular del melanoma son imputables a las 100 mutaciones por Mb y por la heterogeneidad tumoral ${ }^{49}$. La mayor tasa de mutaciones se ha encontrado en melanomas originados en sitios con gran exposición a la luz solar, siendo cinco veces superior en número que en los tumores de piel sin elastosis solar ${ }^{50}$. Adicionalmente, se ha reportado una mayor tasa de mutaciones en tumores BRAF/NRAS silvestres que tienen una mayor complejidad genómica (gran número de transiciones $\mathrm{C}>\mathrm{T}$ ) por radiación $\mathrm{UV}{ }^{52,53}$.

\section{Referencias}

1. Smith AP, Hoek K, Becker D. Whole-genome expression profiling of the melanoma progression pathway reveals marked molecular differences between nevi/melanoma in situ and advanced-stage melanomas. Cancer Biol Ther. 2005;4(9):1018-29.

2. Algazi AP, Soon CW, Daud Al. Treatment of cutaneous melanoma: current approaches and future prospects. Cancer Manag and Res. 2010;2:197-211.

3. Carvajal RD, Antonescu CR, Wolchok JD, Chapman PB, Roman RA, Teitcher J, et al. KIT as a therapeutic target in metastatic melanoma. JAMA. 2011;305(22):2327-34.

4. Chraybi M, Alsamad IA, Copie-Bergman C, Baia M, André J, Dumaz $\mathrm{N}$, et al. Oncogene abnormalities in a series of primary melanomas of the sinonasal tract: NRAS mutations and cyclin D1 amplification are more frequent than KIT or BRAF mutations. Hum Pathol. 2013;44(9):1902-11

5. Grossmann AH, Grossmann KF, Wallander ML. Molecular testing in malignant melanoma. Diagn Cytopathol. 2012;40(6):503-10

6. Kluger HM, Dudek AZ, McCann C, Ritacco J, Southard N, Jilaveanu $L B$, et al. A phase 2 trial of dasatinib in advanced melanoma. Cancer. 2011;117(10):2202-8.

7. Puzanov I, Flaherty KT. Targeted molecular therapy in melanoma. Semin Cutan Med Surg. 2010;29(3):196-201.

8. Bradish JR, Montironi R, Lopez-Beltran A, Post KM, MacLennan GT, Cheng L. Towards personalized therapy for patients with 
malignant melanoma: molecular insights into the biology of BRAF mutations. Future Oncol. 2013;9(2):245-53.

9. Karram S, Novy M, Saroufim M, Loya A, Taraif S, Houreih MA, et al. Predictors of BRAF mutation in melanocytic nevi: analysis across regions with different UV radiation exposure. Am J Dermatopathol. 2013;35(4):412-8.

10. Saroufim M, Habib R, Karram S, Youssef Massad C, Taraif $S$, Loya A, et al. BRAF Analysis on a Spectrum of Melanocytic Neoplasms: An Epidemiological Study Across Differing UV Regions. Am J Dermatopathol. 2013:1-6.

11. Poynter JN, Elder JT, Fullen DR, Nair RP, Soengas MS, Johnson TM, et al. BRAF and NRAS mutations in melanoma and melanocytic nevi. Melanoma Res. 2006;16(4):267-73.

12. Lang J, MacKie RM. Prevalence of exon 15 BRAF mutations in primary melanoma of the superficial spreading, nodular, acral, and lentigo maligna subtypes. J Invest Dermatol. 2005;125(3):575-9.

13. Swick JM, Maize JC Sr. Molecular biology of melanoma. J Am Acad Dermatol. 2012;67(5):1049-54

14. Martí RM, Sorolla A, Yeramian A. New therapeutic targets in melanoma. Actas Dermosifiliogr. 2012;103(7):579-90.

15. Solus JF, Kraft S. Ras, Raf, and MAP kinase in melanoma. Adv Anat Pathol. 2013;20(4):217-26.

16. Mehnert JM, Kluger HM. Driver mutations in melanoma: lessons learned from bench-to-bedside studies. Curr Oncol Rep. 2012;14(5):449-57.

17. Wallander ML, Layfield LJ, Emerson LL, Mamalis N, Davis D, Tripp SR, et al. KIT mutations in ocular melanoma: frequency and anatomic distribution. Mod Pathol. 2011;24(8):1031-5.

18. Postow MA, Hamid O, Carvajal RD. Mucosal melanoma: pathogenesis, clinical behavior, and management. Curr Oncol Rep. 2012;14(5):441-8.

19. Dai $B$, Cai $X$, Kong YY, Yang F, Shen XX, Wang LW, et al. Analysis of KIT expression and gene mutation in human acral melanoma: with a comparison between primary tumors and corresponding metastases/recurrences. Hum Pathol. 2013;44(8):1472-8.

20. Postow MA, Carvajal RD. Therapeutic implications of KIT in melanoma. Cancer J. 2012;18(2):137-41.

21. Minor DR, Kashani-Sabet M, Garrido M, O'Day SJ, Hamid O, Bastian BC. Sunitinib therapy for melanoma patients with KIT mutations. Clin Cancer Res.2012;18(5):1457-63.

22. Tran A, Tawbi HA. Drug evaluation a potential role for nilotinib in KIT-mutated melanoma. Expert Opin Investig Drugs. 2012;4(March 2011):861-9.

23. Chapman PB, Hauschild A, Robert C, Haanen JB, Ascierto P, Larkin J, et al. Improved survival with vemurafenib in melanoma with BRAF V600E mutation. N Engl J Med. 2011;364(26):2507-16.

24. Chapman PB, Hauschild A, Robert C, Larkin J, Haanen J, Ribas $A$, et al. Updated overall survival (OS) results for BRIM-3, a phase III randomized, open-label, multicenter trial comparing BRAF inhibitor vemurafenib (vem) with dacarbazine (DTIC) in previously untreated patients with BRAFV600E-mutated melanoma. J Clin Oncol. 2012;30 (suppl); abstr 8502.

25. Smalley KS, McArthur GA. The current state of targeted therapy in melanoma: this time it's personal. Semin Oncol. 2012;39(2):204-14.

26. Dean E, Lorigan P. Advances in the management of melanoma: targeted therapy, immunotherapy and future directions. Expert Rev Anticancer Ther. 2012;12(11):1437-48.
27. Schlaak M, Bajah A, Podewski T, Kreuzberg N, Von Bartenwerffer W, Wardelmann E, et al. Assessment of clinical parameters associated with mutational status in metastatic malignant melanoma: a single-centre investigation of 141 patients. $\mathrm{Br} \mathrm{J}$ Dermatol.2013:168(4):708-16.

28. Si L, Guo J. C-kit-mutated melanomas: the Chinese experience. Curr Opin Oncol. 2013;25(2):160-5

29. Ashida A, Uhara $H$, Kiniwa $Y$, Oguchi M, Murata $H$, Goto $Y$, et al. Assessment of BRAF and KIT mutations in Japanese melanoma patients. J Dermatol Sci. 2012;66(3):240-2.

30. Jin SA, Chun SM, Choi YD, Kweon SS, Jung ST, Shim HJ, et al. BRAF mutations and KIT aberrations and their clinicopathological correlation in 202 Korean melanomas. J Invest Dermatol. 2013;133(2):579-82.

31. Jung JE, Falk TM, Bresch $M$, Fouto JE, Almut M. BRAF mutations in cutaneous melanoma: no correlation with histological prognostic factors or overall survival. J Bras Patol Med Lab 2010;46(6):487-493.

32. Mazzei ME, Hochmann J, Manrique G, Mariño AL, Delgado L, Martínez-Asuaga M. Determinación de la mutación BRAF V600E en melanomas de pacientes uruguayos. Rev Méd Urug 2013;29(2):1-6

33. Delgadillo DA. Identificación del oncogén BRAF en pacientes mexicanos con melanoma lentiginoso acral [tesis de grado]. Instituto Politécnico Nacional.

34. <http://www.cancerstaging.org/staging/posters/ melanoma8.5x11.pdf>

35. Bauer J, Büttner P, Murali R, Okamoto I, Kolaitis NA, Landi MT, et al. BRAF mutations in cutaneous melanoma are independently associated with age, anatomic site of the primary tumor, and the degree of solar elastosis at the primary tumor site. Pigment Cell Melanoma Res. 2011;24(2):345-51.

36. Liu W, Kelly JW, Trivett M, Murray WK, Dowling JP, Wolfe R, et al. Distinct clinical and pathological features are associated with the BRAF(T1799A(V600E)) mutation in primary melanoma. J Invest Dermatol. 2007;127(4):900-5

37. Omholt K, Platz A, Kanter L, Ringborg U, Hansson J. NRAS and BRAF mutations arise early during melanoma pathogenesis and are preserved throughout tumor progression. Clin Cancer Res. 2003:9(17):6483-8

38. Long GV, Menzies AM, Nagrial AM, Haydu LE, Hamilton AL, Mann GJ, et al. Prognostic and clinicopathologic associations of oncogenic BRAF in metastatic melanoma. J Clin Oncol. 2011;29(10):1239-46.

39. Chapman PB, Hauschild A, Robert C, Haanen JB, Ascierto $P$, Larkin J, et al. Improved survival with vemurafenib in melanoma with BRAF V600E mutation. N Engl J Med. 2011;364(26):2507-16.

40. Menzies AM, Haydu LE, Visintin L, Carlino MS, Howle JR, Thompson JF, et al. Distinguishing clinicopathologic features of patients with V600E and V600K BRAF-mutant metastatic melanoma. Clin Cancer Res. 2012;18(12):3242-9.

41. Jakob JA, Bassett RL, Ng CS, Curry JL, Joseph RW, Alvarado GC, et al. NRAS mutation status is an independent prognostic factor in metastatic melanoma. Cancer. 2012;118(16):4014-23.

42. Lee JH, Choi JW, Kim YS. Frequencies of BRAF and NRAS mutations are different in histological types and sites of origin of cutaneous melanoma: a meta-analysis. $\mathrm{Br} J$ Dermatol. 2011;164(4):776-84 
43. Ellerhorst JA, Greene VR, Ekmekcioglu S, Warneke CL, Johnson MM, Cooke CP, et al. Clinical correlates of NRAS and BRAF mutations in primary human melanoma. Clin Cancer Res. 2011;17(2):229-35.

44. Devitt B, Liu W, Salemi R, Wolfe R, Kelly J, Tzen CY, et al. Clinical outcome and pathological features associated with NRAS mutation in cutaneous melanoma. Pigment Cell Melanoma Res. 2011;24(4):666-72.

45. Kong $Y$, Si L, Zhu $Y$, Xu X, Corless $C L$, Flaherty $K T$, et al. Largescale analysis of KIT aberrations in Chinese patients with melanoma. Clin Cancer Res.2011;17(7):1684-91.

46. Omholt K, Grafström E, Kanter-Lewensohn L, Hansson J, Ragnarsson-Olding BK. KIT pathway alterations in mucosal melanomas of the vulva and other sites. Clin Cancer Res. 2011;17(12):3933-42.

47. Woodman SE, Lazar AJ, Aldape KD, Davies MA. New strategies in melanoma: molecular testing in advanced disease. Clin Cancer Res. 2012;18(5):1195-200.

48. Hill VK, Gartner JJ, Samuels Y, Goldstein AM. The genetics of melanoma: recent advances. Annu Rev Genomics Hum Genet. 2013;14:257-79.

49. De Vries E, Nijsten TEC, Visser O, Bastiaannet E, van Hattem $\mathrm{S}$, Janssen Heijnen $\mathrm{ML}$, et al. Superior survival of females among 10,538 Dutch melanoma patients is independent of
Breslow thickness, histologic type and tumor site. Ann Oncol. 2008; 19(3):583-9.

50. Lawrence MS, Stojanov P, Polak P, Kryukov GV, Cibulskis $\mathrm{K}$, Sivachenko $\mathrm{A}$, et al. Mutational heterogeneity in cancer and the search for new cancer-associated genes. Nature. 2013;499(7457):214-8.

51. Curtin JA, Busam K, Pinkel D, Bastian BC. Somatic activation of KIT in distinct subtypes of melanoma. J Clin Oncol. 2006;24(26):4340-6.

52. Hodis E, Watson IR, Kryukov GV, Arold ST, Imielinski M, TheuriIlat JP, et al. A landscape of driver mutations in melanoma. Cell. 2012;150(2):251-63.

53. Mar VJ, Wong SQ, Li J, Scolyer RA, McLean C, Papenfuss AT, et al. BRAF/NRAS wild-type melanomas have a high mutation load correlating with histological and molecular signatures of UV damage. Clin Cancer Res. 2013;19(17):4589-98.

54. Vogelstein B, Papadopoulos N, Velculescu VE, Zhou S, Diaz LA Jr, Kinzler KW. Cancer genome landscapes. Science. 2013;339(6127):1546-58.

55. Joosse A, Collette S, Suciu S, Nijsten T, Lejeune F, Kleeberg UR, et al. Superior outcome of women with stage $1 / /$ l cutaneous melanoma: pooled analysis of four European Organisation for Research and Treatment of Cancer phase III trials. J Clin Oncol. 2012;30(18):2240-7. 